\title{
BARREIRAS NA COMUNICAÇÃO E ALTERNATIVAS PARA AUXILIAR A COMPREENSÃO SOBRE AS MUDANÇAS CLIMÁTICAS
}

\author{
M. M. GUARENGHI ${ }^{1 *}$, M. de A. AZEVEdo ${ }^{1}$, A. WALTER $^{1}$, C. K. N. CAVALIERO ${ }^{1}$ \\ ${ }^{1}$ Planejamento de Sistemas Energéticos/Faculdade de Engenharia Mecânica, Universidade Estadual de Campinas \\ (UNICAMP) \\ marjorie@fem.unicamp.br \\ Submetido 11/05/2016 - Aceito 30/06/2018 \\ DOI: $10.15628 /$ holos.2018.5322
}

\section{RESUMO}

O entendimento das causas e riscos das mudanças climáticas e das ações para minimizar os problemas associados a essas alterações é fundamental para conscientizar a sociedade sobre a importância desse aspecto ambiental. As informações sobre as mudanças climáticas têm aumentado nos últimos anos. Porém, a comunicação enfrenta desafios em traduzir o conhecimento científico em uma linguagem não acadêmica e de fácil assimilação para a população. Além disso, a polarização e politização da ciência climática e a dificuldade em motivar a sociedade a mudar seu estilo de vida a fim de reduzir impactos ambientais são importantes questões a serem consideradas. Este trabalho apresenta os principais fatores que limitam a comunicação eficaz sobre as mudanças climáticas e mostra algumas alternativas para melhorar a comunicação entre a ciência e a sociedade, estimulando-a a agir de forma mais participativa a fim de mitigar o aquecimento global.

PALAVRAS-CHAVE: mudanças climáticas, comunicação ambiental, mídia.

\section{BARRIERS IN COMMUNICATION AND ALTERNATIVES TO SUPPORT UNDERSTANDING ON CLIMATE CHANGE}

\begin{abstract}
The understanding of the causes and risks of climate change, as well as the knowledge on how to mitigate the problems associated with this issue, is fundamental to make the society aware of the importance of this environmental aspect. Information about climate change has increased in the past years. However, the communication is facing challenges in translating the scientific knowledge into a non-academic language easy to assimilate. Also, the politicization and polarization of
\end{abstract}

climate science and the difficulty in motivating society to change their lifestyle in order to reduce environmental impacts are important issues to be considered. This paper addresses the main factors that limit the communication effectiveness on climate change, and presents alternatives to improve the communication between science and society and thus engage it to mitigate the global warming.

KEYWORDS: climate change, environmental communication, media. 


\section{INTRODUÇÃO}

As mudanças climáticas consistem em uma das principais preocupações ambientais expondo pessoas, sociedade, setores econômicos e ecossistemas a riscos (IPCC, 2014). Dentre algumas evidências das mudanças climáticas apresentadas no quinto relatório do Painel Intergovernamental de Mudanças Climáticas - IPCC, destaque é dado ao aumento da temperatura atmosférica, ao aquecimento das águas nos oceanos, ao aumento do nível do mar, à redução nas camadas de gelo e à acidificação dos oceanos (IPCC, 2014).

Para prevenir e minimizar as mudanças climáticas é necessário entender a extensão de suas ameaças e oportunidades de mitigação, e assim desenvolver a capacidade de projetar as condições futuras do planeta e compreender as vulnerabilidades causadas pelos impactos globais. Nesse contexto, estabelece-se a importância da comunicação sobre o tema em diversas escalas, da instauração de políticas públicas e das reuniões internacionais anuais, como a das Conferências das Partes (COP), a fim de engajar as nações nos esforços de mitigação da emissão de gases de efeito estufa.

Trabalhar a percepção pública sobre as alterações climáticas e seus riscos é crucial para que os países colaborem nas mudanças de hábitos necessárias (Moser, 2016; Bolsen, Druckman \& Cook, 2015). A mídia pode exercer fundamental participação na eficácia dessa comunicação, envolvendo as partes através de informações de qualidade a respeito dos riscos ambientais, avanços na ciência, notícias do mundo, novas políticas e projetos inovadores. Porém, a maneira como as notícias sobre as mudanças climáticas vêm sendo transmitidas ao público tem contribuído para o atraso de ações.

O IPCC é o principal órgão compilador dos estudos sobre mudanças climáticas atuando há mais de 20 anos. Os Assessment Report do IPCC concentram resultados e análises de especialistas de diversas áreas sobre as causas, os riscos e impactos das mudanças climáticas, estimativas de mudanças do clima futuras, e caminhos sobre adaptações, mitigação e desenvolvimento sustentável (IPCC, 2014).

Para prever cenários futuros, os cientistas envolvidos se utilizam de uma combinação de observações e modelos probabilísticos. Com essas informações, pretende-se fornecer subsídios para governos, para o setor privado e para o público, a fim de que se aja o mais rapidamente com relação à mitigação das emissões de gases de efeito estufa e, consequente, ao aquecimento global associado às ações antrópicas.

O futuro do clima depende muito de fatores difíceis de serem contabilizados e estimados, como políticas públicas e ações que vão além das práticas científicas. O público tem acesso às informações sobre o clima por diversas fontes, diferentes do IPCC e de outros relatórios científicos. No entanto, esses outros meios de comunicação, por muitas vezes, fornecem dados confusos e parcialmente corretos, ou até mesmo tendenciosos (Stoutenborough, Fette, Vedlitz \& Goldsmith, 2014).

A situação atual do planeta exige ações imediatas, contínuas e com maior potencial de alcance. A energia consumida deverá vir de fontes mais limpas, o uso energético deverá ser mais eficiente, a população deverá se adaptar a hábitos cotidianos menos energo-intensivos e a uma vida mais sustentável (IPCC, 2007). Educar é algo amplo e global (Silva, 2015; Jacobi, Guerra, 
Sulaiman \& Nepomuceno, 2011). Na era da internet e redes sociais, o ensino vai além da escola e é altamente influenciado pela mídia que aponta e forma comportamentos. O resultado revela-se numa percepção distante e abstrata sobre as mudanças climáticas, que não condizem com a urgência da causa.

Tendo em vista a importância da ampla e adequada divulgação sobre as mudanças climáticas, o objetivo deste trabalho é apresentar as principais dificuldades para se ter uma comunicação eficaz sobre as mudanças climáticas, além de mostrar maneiras de como encaminhar e auxiliar a comunicação referente à essas alterações a fim de conscientizar e engajar diversos públicos a mudarem seus hábitos e mitigar os impactos antrópicos sobre o clima.

\section{BARREIRAS NA COMUNICAÇÃO, COMPREENSÃO E ENGAJAMENTO PÚBLICO RELACIONADOS ÀS MUDANÇAS CLIMÁTICAS}

Diversos são os fatores que influenciam a opinião pública sobre as preocupações com as consequências das mudanças climáticas. Em uma revisão realizada por Brulle, Carmickael e Jenkins (2012), estudos mostram que a opinião pública sobre o tema é influenciada:

a) por diversos eventos climáticos extremos, como ondas de calor e frio com temperaturas extremas, ocorrência de furacões, inundações e secas;

b) pela falta da divulgação da informação científica, que contribui para a dificuldade de compreensão do tema;

c) por movimentos ou grupos denominados denier formado por céticos quanto às mudanças climáticas, ou por movimentos defensores da questão, além de blogs, sites e projetos da mídia;

d) pela ideologia política dos cidadãos;

e) por fatores econômicos, como baixa renda e nível de desemprego, vistos como prioritários frente à questão climática;

f) pela cobertura da mídia; e

g) pela falta de orientação educacional direcionada para as mudanças climáticas (Silva, 2015).

Nos últimos anos, têm sido observados avanços na conscientização sobre as mudanças climáticas, no apoio a políticas climáticas e de energia limpa, e até mesmo na compreensão de como melhorar a comunicação e educação sobre as mudanças climáticas. No entanto, persistem alguns desafios a serem enfrentados, como a compreensão superficial do público sobre o tema, a transição da consciência e preocupação para a ação, a comunicação em ambientes polarizados e politizados, além de outros fatores que dificultam a comunicação eficaz sobre as questões ambientais de natureza climática e os riscos associados a estas (Moser, 2016). A seguir são apresentadas algumas das principais dificuldades relacionadas à comunicação sobre as mudanças climática.

\subsection{Comunicação das pesquisas científicas sobre mudanças climáticas}

A percepção do público ao aquecimento global é influenciada pelas notícias de base científica e ambiental (Zhao, Leiserowitz, Maibach \& Roser-Renouf, 2011). Avanços no conhecimento científico relacionados ao aprimoramento de modelos climáticos, com análises 
mais sofisticadas, maior disponibilidade de dados em regiões até então não monitoradas permitindo melhores estimativas sobre riscos e reduzindo incertezas (IPCC, 2014) têm contribuído para fornecer mais informações a serem divulgadas à sociedade sobre diversos tópicos relacionados ao tema.

No entanto, as publicações científicas geralmente são complexas e traduzir o conhecimento científico em uma linguagem não-técnica e de fácil assimilação ao público não é simples. Apesar de muitos avanços na ciência climática, a dificuldade em repassar as informações existentes contribui para lacunas no entendimento público e político sobre as causas, riscos e ações de mitigação das mudanças climáticas (Moser, 2016; Stoutenborough et al., 2014; Bolsen et al., 2014; Lorenzoni \& Pidgeon, 2006).

Para tanto é necessário que haja um contato maior entre os cientistas e a mídia que, por meio da televisão e dos jornais, em geral, é a principal fonte primária de informação. Em entrevistas feitas com mais de 400 cientistas climáticos norte-americanos com publicações em periódicos, Stoutenborough et al. (2014) relatam que, em geral, os pesquisadores não acreditam que a cobertura da mídia sobre mudanças climáticas globais seja precisa. Muitos afirmam que suas pesquisas estão sendo divulgadas na mídia, mas que a mídia tem pouco contato direto com os cientistas. A mídia recorre a trabalhos em periódicos e a estudos publicados e, a falta de compreensão dos atores da mídia a esses materiais, por não serem especialistas em mudanças climáticas, faz com que resultados sejam reportados de forma imprecisa ou muito resumidos. Por outro lado, observou-se também que os cientistas não são proativos em buscar a mídia para divulgar suas descobertas, tampouco para corrigir informações divulgadas de forma equivocada.

As notícias, em geral, possuem caráter informativo, porém, estudos mostram que a cobertura de notícias com maior contexto científico, maiores detalhes explicativos auxiliam na compreensão do público sobre mudanças climáticas (Akerlof, Rowan, Fitzgerald \& Cedeno, 2012). Porém, a dificuldade financeira da mídia reduz o jornalismo cientifico, diminuindo a presença de especialistas vinculados à comunicação que possam melhor compreender e reportar aspectos das ciências climáticas (Akerlof et al., 2012).

\subsection{Polarização ideológica}

De forma geral, existe consenso científico de que o planeta passa atualmente por um período de aquecimento (Cook et al., 2013). As mudanças climáticas são explicadas por forçamentos radioativos naturais (como as atividades solar e vulcânica) e antropogênicos, e, conforme apresentado nos relatórios do IPCC de forma crescente é impossível justificar o aquecimento global das últimas décadas do século XX apenas pelos forçamentos naturais (IPCC, 2007; IPCC, 2014). No entanto, alguns poucos cientistas céticos negam que o planeta esteja passando por um período de aquecimento (Singer, 2011), afirmam que a influência humana sobre o clima é pouco provável e dificilmente seria detectada (Molion, 2008); outros acreditam que o aquecimento é uma realidade, mas não atribuem suas causas à atividade antrópica (Abdussamatov, 2013), e também há os que concordam com a influência antrópica sobre o aquecimento, porém afirmam que as consequências futuras apresentadas são exageradas, como Lomborg (2011).

Em contrapartida às mensagens de risco e impacto das mudanças climáticas, também há um pequeno número de cientistas legitimados que focam nas incertezas sobre eventos climáticos 
futuros, suas consequências para o bem-estar da humanidade (Weber \& Stern, 2011) e, na imprecisão dos modelos climáticos em prever tais impactos futuros (Stoutenborough et al.,2014; Molion, 2008). Contribuem também com a crítica às ciências climáticas, organizações e movimentos contrários ao consenso científico, muitas vezes patrocinados por grandes companhias de petróleo e gás (Weber \& Stern, 2011; Posner, 2004).

Dessa forma, tanto cientistas céticos, como organizações contrárias às mudanças climáticas geram um discurso polarizado sobre o tema. A polarização ideológica influencia na confiança das pessoas na ciência, nas atitudes individuais sobre mudanças climáticas e nas escolhas sobre comportamentos relacionados à eficiência energética (Farrell, 2015).

Analisando textos com viés contrário às mudanças climáticas entre 1993 e 2013 nos Estados Unidos (incluindo artigos científicos, textos de blogs, artigos na internet, declarações políticas e artigos da imprensa), Farrell (2015) demostrou que o financiamento corporativo influencia o conteúdo temático de um discurso polarizador. As organizações que receberam financiamento corporativo mostraram maior probabilidade de terem seus textos escritos e divulgados. Grupos de movimentos denier criam táticas junto às empresas e organizações a fim de gerar dúvidas sobre o consenso científico e a necessidade de tomar medidas para resolver a questão (Brulle et al., 2012), afetando o entendimento do público e dos tomadores de decisão com relação aos riscos das mudanças climáticas (Stoutenborough et al.,2014).

\subsection{Politização das questões climáticas}

Baseando-se em todas as pesquisas nacionais dos Estados Unidos disponíveis entre 2002 e 2010 sobre a preocupação dos norte-americanos com as mudanças climáticas, Brulle et al. (2012) identificaram que o fator de maior influência sobre a opinião pública a respeito do assunto era a batalha partidária sobre as mudanças climáticas (declarações de partidos políticos, votações sobre o clima e audiências no Congresso sobre o tema). Dentre outros quatro fatores analisados, movimentos pró ou contra o tema e a cobertura da mídia foram considerados relevantes, enquanto a ocorrência de eventos climáticos extremos e o acesso público a informações científicas precisas tiveram efeito mínimo sobre as opiniões públicas.

A politização da questão climática nos Estados Unidos teve início nos anos 90 para confrontar o consenso científico sobre a ciência do clima, motivada por interesses de indústrias de combustíveis fósseis e por pensadores alinhados a interesses comerciais. Esse fato reduziu a confiança dos conservadores em cientistas não-céticos quanto às mudanças climáticas e contribuiu para a polarização da questão (Bolsen et al., 2016).

A intensa influência da ideologia política sobre a forma dos cidadãos norte-americanos pensarem sobre mudanças climáticas é preocupante. Esta interfere na conscientização sobre os problemas das mudanças climáticas e, consequentemente, no engajamento às ações de mitigação dos gases de efeito estufa. Os americanos representam uma parcela da população mundial cujas atividades contribuem significativamente para o aquecimento global, visto que os EUA são o segundo maior emissor de gases de efeito estufa (WRI, 2015). 


\subsection{A lacuna entre a conscientização e a ação.}

Em uma pesquisa feita nos Estados Unidos e no Reino Unido, apesar da percepção do público sobre as mudanças climáticas estar aumentando, o nível de preocupação declinou nos últimos anos (Spence, Poortinga \& Pidgeon, 2012). Parte do motivo para essa falta de interesse vem da dificuldade que as pessoas possuem em mudar seus comportamentos em prol de exercerem hábitos mais sustentáveis. Isso se deve a exigência da adaptação a uma rotina mais rígida. Watson et al. (2016) sugerem que a maioria das pessoas ainda assimilam o assunto como algo abstrato, psicologicamente distante e até mesmo controverso.

Uma pesquisa indicou que tradicionalmente as pessoas tendem a enxergar os riscos climáticos como uma questão que só irá afetar outras nações e/ou gerações futuras. Ao se comparar benefícios futuros e benefícios em curto prazo, a tendência é que o futuro se torne particularmente pouco atraente, pois necessita de ações imediatas com recompensas muito distantes no tempo (Spence et al., 2012). Sendo assim, as mudanças climáticas passam a receber tratamento secundário, principalmente diante de outros problemas (Lorenzoni \& Pidgeon, 2006).

Segundo Brulle et al. (2012), para uma parcela do público, as opiniões a respeito das mudanças climáticas são estáveis e altamente associadas a princípios sociais e ideológicos. A mídia, por sua vez, é um dos segmentos descritos com o poder de promover mudanças de opinião dentre a população. Um crescente número de estudos mostra como a informação trabalhada na forma de diálogo é capaz de favorecer o conhecimento mais intenso, a empatia das pessoas, a mudança de atitudes e a receptividade a novas políticas alternativas, bem mais do que a informação que é apenas transmitida (Moser, 2016).

Outro ponto importante é a grande influência de fatores políticos e econômicos sob a posição pública (Brulle et al., 2012), como, por exemplo, o aumento em taxas de desemprego e a redução na renda per capita (Kahn \& Kotchen, 2010). Estes fatores impactam negativamente na implementação de políticas ambientais. A fim de se trabalhar a ação pública mesmo diante de variáveis independentes, é necessário analisar cuidadosamente seus efeitos de interferência nas pessoas.

\section{POSSIBILIDADES DE MELHORIA NA COMUNICAÇÃO SOBRE MUDANÇAS CLIMÁTICAS}

Uma comunicação efetiva que motive o engajamento público em prol da mitigação das mudanças climáticas depende do entendimento e conscientização sobre suas causas, riscos e potenciais formas de minimizar o problema. Com o objetivo de auxiliar a comunicação referente à natureza abstrata das ciências climáticas, à informação de base científica, especialistas apontam o uso de tecnologias de comunicação e informação para criar imagens visuais (Ballantyne, Wibeck \& Neset, 2016; Wozniak, Lück \& Wessler, 2015; Sheppard, 2012). Dentre as formas de visualização, podem ser citados gráficos de computadores avançados, visualização de paisagens 3D e ambientes imersivos como planetários digitais que auxiliem na explicação das causas e consequências das mudanças climáticas (Ballantyne et al., 2016).

Tais tecnologias de comunicação e informação são ferramentas importantes a serem empregadas pela mídia tradicional representada pelos jornais e programas televisivos. Com a maior diversidade de canais e programas sobre assuntos específicos, criam-se oportunidades 
para um jornalismo científico que possua conteúdo mais explicativo do que informativo. Alguns estudos sugerem que a cobertura de notícias científicas com detalhes mais explicativos, como informações obtidas a partir de modelos climáticos, auxilia na compreensão do público, reduzindo incertezas e dúvidas relacionadas às mudanças climáticas (Akerlof et al., 2012; Corbett \& Durfee, 2004).

Estratégias de comunicação apresentam eficácia variada para públicos distintos e pode ser vantajoso separar e direcionar mensagens para pessoas com visões diferentes (Xue et al., 2016). Bostrom, Böhm e O’Connor (2013) mostram que estudos utilizando estratégias de marketing social de identificação de grupos específicos (targeting) e individualização da informação, personalizando-a dentro de um design de comunicação (tailoring) e adaptando-a para públicos específicos, foram eficazes na educação sobre mudanças climáticas e suporte a decisões. Segundo Bostrom et al. (2013), tais estratégias têm sido utilizadas para encorajar comportamentos pró-ambientais em vários focos, incluindo a redução do consumo energético.

Para se ter uma comunicação bem-sucedida sobre as mudanças climáticas, pesquisadores têm realizado entrevistas com diferentes grupos focais, estudado o papel dos valores, crenças e visões do mundo, analisado mudanças de percepção, compreensão, atitudes do público, e avaliado como a comunicação sobre o tema é (ou deve ser) feita para diferentes grupos focais (Moser, 2016). De forma sucinta, a Tabela 1 apresenta alguns resultados de autores, como Stoknes (2015), Marshall (2014) e Washington \& Cook (2011), que têm estudado sobre as defesas psicológicas das pessoas com relação ao tema das mudanças climáticas como: não atribuir devida importância; não pensar sobre o assunto; ou não se sentir parte do problema. Os pesquisadores mostram como as pessoas com características relativas a cada grupo encaminham a comunicação do tema, e sugerem alternativas mais adequadas para atingir pessoas com diferentes tipos de opinião sobre as mudanças climáticas, conscientizando-as e educando-as a fim de engajá-las a agir para mitigar a influência antrópica sobre o clima.

Uma das estratégias para melhorar a comunicação sobre mudanças climáticas para pessoas que não possuem conhecimento sobre o tema e ainda não sentem seus efeitos (primeiro grupo apresentado na Tabela 1) consiste em dar uma percepção em escala local das mudanças climáticas para essas pessoas, mostrando potenciais riscos que as mudanças climáticas podem trazer para lugares próximos de onde essas pessoas vivem. Em um contexto semelhante, Hu e Chen (2016) implementaram um programa educacional com adolescentes em áreas rurais da China no qual estudantes de 10 a 13 anos comunicaram-se com moradores locais, com mais de 60 anos, sobre as mudanças no clima observadas por eles. As lembranças dos antigos moradores estavam de acordo com o histórico meteorológico da região. O grupo que assistiu a uma aula sobre mudanças climáticas, seguida do contato com os idosos tiveram menos incertezas, maior preocupação e percepção do risco, se sentiram mais intencionados a mitigar as mudanças do que os grupos que apenas assistiram à aula, ou que simplesmente não tiveram qualquer instrução sobre o assunto. 
Tabela 1: Defesas psicológicas e respectivas formas de como a comunicação sobre mudanças climáticas pode ser encaminhada.

\begin{tabular}{|c|c|c|c|}
\hline Defesas & $\begin{array}{l}\text { Ação de comunicação dos "pró- } \\
\text { clima" }\end{array}$ & Ação de comunicação dos "anti-clima" & Abordagem para melhorar a comunicação \\
\hline $\begin{array}{l}\text { Defesa quanto ao pensar, } \\
\text { sentir e saber sobre } \\
\text { mudanças climáticas: } \\
\text { Manter distância psicológica } \\
\text { da questão (temporal, } \\
\text { espacial, conceitual/ } \\
\text { perceptiva), evitando-a, } \\
\text { focando no presente, } \\
\text { distraindo-se, tendo viés } \\
\text { otimista }\end{array}$ & $\begin{array}{l}\text { Enfatizam a mudança climática } \\
\text { como uma questão da ciência; } \\
\text { Usam linguagem ou gráficos } \\
\text { científicos abstratos; } \\
\text { Falam sobre as mudanças climáticas } \\
\text { como uma ameaça futura em } 100 \\
\text { anos; } \\
\text { Usam imagens de eventos em } \\
\text { lugares distantes, derretimento das } \\
\text { placas de gelo }\end{array}$ & $\begin{array}{l}\text { Não falam sobre ou limitam notícias } \\
\text { sobre o assunto; } \\
\text { Enfatizam a naturalidade da } \\
\text { variabilidade do clima e suas } \\
\text { mudanças; Evitam o "aquecimento } \\
\text { global"; } \\
\text { "Por que se preocupar agora? Há } \\
\text { assuntos mais urgentes para focar. Isso } \\
\text { não vai acontecer aqui. Nós podemos } \\
\text { lidar com isso, vamos ficar bem." }\end{array}$ & $\begin{array}{l}\text { Trazer a questão para o presente, } \\
\text { aproximando-a das pessoas, mostrando seu } \\
\text { caráter humano, pessoal, e urgente; } \\
\text { Falar de pessoas, lugares e entidades } \\
\text { próximos de onde as pessoas alvo estão; } \\
\text { Usar a emoção e histórias para dar um } \\
\text { significado; } \\
\text { Envolver as pessoas diretamente no } \\
\text { controle de alterações; }\end{array}$ \\
\hline $\begin{array}{l}\text { Defesa contra o pensar sobre } \\
\text { ou sentir a perda associada } \\
\text { com mudanças climáticas: } \\
\text { Perder a aversão/senso do } \\
\text { medo/desgraça levando a } \\
\text { evitar a questão, não agir, } \\
\text { transferir a responsabilidade } \\
\text { para outros/futuro }\end{array}$ & $\begin{array}{l}\text { Usam quadros de perdas e de } \\
\text { custos; } \\
\text { Usam quadros de catástrofe, } \\
\text { imagens de desastres, imagens ou } \\
\text { histórias apocalípticas; } \\
\text { Não ligam riscos a ações }\end{array}$ & $\begin{array}{l}\text { Fazem uso de eufemismos, } \\
\text { reformulando negativo como positivo; } \\
\text { Enfatizam os desastres como sendo de } \\
\text { causa natural; } \\
\text { Jogam com o medo das soluções ao } \\
\text { invés do medo do problema; } \\
\text { Destacam como seria caro mudar a } \\
\text { partir do status que estamos }\end{array}$ & $\begin{array}{l}\text { Concentrar-se em perdas passadas e } \\
\text { restauração do que foi perdido; concentrar- } \\
\text { se em benefícios e oportunidades em curto } \\
\text { prazo para evitar perdas ou custos futuros; } \\
\text { Usar mensagens de engajamento (como } \\
\text { fazer o que é certo, prontidão, segurança); } \\
\text { Focar na melhoria da saúde e qualidade de } \\
\text { vida (pessoas, família, comunidade); }\end{array}$ \\
\hline $\begin{array}{l}\text { Defesa contra a culpa; sem } \\
\text { ligar o conhecimento à ação: } \\
\text { Lidar com a dissonância } \\
\text { cognitiva por meio da dúvida } \\
\text { da ciência, minimizando o } \\
\text { problema, diminuindo } \\
\text { soluções, evitando a questão, } \\
\text { não agindo, transferindo } \\
\text { responsabilidade para outros }\end{array}$ & $\begin{array}{l}\text { Usam de recursos explícitos ou } \\
\text { implícitos de culpa; } \\
\text { Exageram o estado da ciência } \\
\text { (evitam falar das incertezas) }\end{array}$ & $\begin{array}{l}\text { Jogam com incertezas científicas para } \\
\text { justificar a falta de ação; } \\
\text { Questionam a integridade dos } \\
\text { cientistas; } \\
\text { Confundem as pessoas (por exemplo, o } \\
\mathrm{CO}_{2} \text { é uma substância natural } \\
\text { necessário para a vida, como pode ser } \\
\text { ruim?) }\end{array}$ & $\begin{array}{l}\text { Enfatizar o alto grau de consenso científico; } \\
\text { Justificar a ação motivada pelas incertezas; } \\
\text { Melhor prevenir do que remediar; } \\
\text { Enfatizar a causa moral para a ação } \\
\text { climática; } \\
\text { Oferecer oportunidades para ação clara, } \\
\text { coerente e visível }\end{array}$ \\
\hline
\end{tabular}

Fonte: Adaptado de Moser (2016), com base em Stoknes (2015), Marshall (2014) e Washington \& Cook (2011).

HOLOS, Ano 34, Vol. 03 
A comunicação sobre as mudanças climáticas também deve ser utilizada para despertar a mobilização social. No Reino Unido, uma campanha denominada The Student Switch Off (SSO, 2016) incentiva alunos ingressantes em diversas universidades a mudar seus hábitos de vida, estimulando-os a adotar medidas para economizar energia diariamente. A campanha baseia-se no pressuposto de que a conscientização sobre o uso racional de energia é um marco para os jovens que, ao ingressar na universidade, iniciam uma nova e importante etapa em suas trajetórias.

Em cada universidade que adere à campanha, os alunos são incentivados a poupar energia e encontrar medidas para reduzir o consumo energético, sem qualquer incentivo financeiro. São realizadas competições com prêmios de incentivo e atividades para criar senso comunitário. Técnicas de marketing visual em mídias sociais como postagem de fotos em páginas do Facebook e vídeos no Youtube, tanto das competições, quanto das medidas de conscientização, são utilizadas para divulgar mensagens de segurança energética (O'Neill \& Boykoff, 2011). A campanha já se espalhou por universidades da Suíça, Grécia, Chipre e Lituânia. No último ano letivo (2015/2016), os estudantes da campanha reduziram em média 6,6\% do consumo de energia elétrica nos corredores das residências universitárias dos alunos que aderiram à campanha, deixando de emitir 1.800 toneladas de $\mathrm{CO}_{2}$ para a atmosfera $(\mathrm{SSO}, 2016)$.

A educação visando promover uma maior consciência ambiental e propondo mudanças comportamentais a fim de promover o desenvolvimento sustentável é fundamental para contribuir com o engajamento da sociedade a fim de minimizar as mudanças climáticas (Silva, 2015; Hu \& Chen, 2016; Bostrom et al., 2013). No entanto, são necessário investimentos em projetos educacionais (Silva, 2015).

No Brasil, a importância da educação ambiental nas escolas começou a ser representativa nos anos 2000 com a criação do Órgão Gestor (OG) da Política Nacional de Meio Ambiente (PNMA) (Jacobi et al., 2011). Desde então, são realizados encontros com educadores ambientais visando debater a interface entre educação ambiental e mudanças climáticas (Jacobi et al., 2011). Como exemplo, pode-se citar a "Oficina sobre Educação Ambiental no contexto das Mudanças Climáticas" realizada em 2009 pelo OG. A Oficina contou com membros de universidades, instituições governamentais e outros convidados que propuseram orientações para ações nacionais de educação ambiental (BRASIL, 2011). Como resultado de uma ação coordenada pelo Departamento de Educação Ambiental e o Ministério do Meio Ambiente, foi lançado em 2013 o documento "Educação ambiental \& Mudanças Climáticas". A publicação visa apresentar dados, informações e análises sobre a relação entre educação ambiental e mudanças climáticas a fim de estimular a reflexão, ação e elaboração de políticas públicas que possam contribuir para abordar o tema de uma forma crítica e transformadora (BRASIL, 2013).

\section{CONCLUSÕES}

O reconhecimento dos argumentos dados pela comunidade científica sobre as mudanças no clima e as evidencias de alterações que já estão ocorrendo ressaltam a seriedade e a urgência com que a questão deve ser abordada. 
A sociedade e tomadores de decisão dependem da comunicação sobre a questão climática para compreender as causas, os riscos, as vulnerabilidades e despertar a percepção sobre as necessidades de mitigação e adaptação decorrentes desse problema ambiental. É necessário que o tema seja priorizado na esfera pública, que o conhecimento científico seja traduzido de maneira clara para diferentes públicos, que as barreiras existentes na interface entre comunicação e mudanças climáticas sejam contornadas a fim de esclarecer dúvidas existentes e conscientizar as pessoas da influência antrópica no agravamento das mudanças do clima.

Para tanto, destacam-se as mudanças nas estratégias de comunicação, como as técnicas de marketing social em direcionar e adaptar informações para públicos específicos, o uso de tecnologias de comunicação e informação para criar imagens visuais, e também o marketing visual em mídias sociais. As análises dos valores, das visões do mundo, da compreensão da percepção de diferentes públicos quanto às mudanças climáticas também têm sido apontadas para sugerir formas de abordagem de comunicação mais eficazes a fim conscientizar e mobilizar a sociedade para agir em prol da mitigação da atividade antrópica sobre o clima.

\section{REFERÊNCIAS}

Abdussamatov, H. I. (2013). Grand minimum of the total solar irradiance leads to the little ice age. Journal of Geology \& Geophysics, 2(113). doi: 10.4172/2329-6755.1000113.

Akerlof, K., Rowan, K. E., Fitzgerald, D., \& Cedeno, A. Y. (2012). Communication of climate projections in US media amid politicization of model science. Nature Climate Change, 2(9), 648-654. doi: 10.1038/NCLIMATE1542.

Ballantyne, A. G., Wibeck, V., Neset, T-S. (2015). Images of climate change - a pilot study of young people's perceptions of ICT- based climate visualization. Climatic Change, 134, 73-85. doi: 10.1007/s10584-015-1533-9.

Bolsen, T., Druckman, J. N., \& Cook, F. L. Citizens', scientists', and policy advisors' beliefs about global warming. (2015). The ANNALS of the American Academy of Political and Social Science, 658(1), 271-295. doi: 10.1177/0002716214558393.

Bostrom, A., Böhm, G., \& O'Connor, R.E. (2013). Targeting and tailoring climate change communication to audiences. WIREs Climate Change, 4(5), 447-455. doi: 10.1002/wcc.234.

BRASIL. (2011). Departamento de Educação Ambiental - Balanço de 2009/2010. Ações realizadas $e$ em andamento. Brasília: Ministério do Meio Ambiente/Secretaria de Articulação Institucional e Cidadania Ambiental/ Departamento de Educação Ambiental.

BRASIL. (2013). Educação ambiental \& Mudanças Climáticas. Diálogo necessário num mundo em transição. Série educativa. Brasília: Ministério do Meio Ambiente/Secretaria de Articulação Institucional e Cidadania Ambiental/ Departamento de Educação Ambiental. Brasília. Recuperado de www.mma.gov.br/informma/item/8266.

Brulle, R. J., Carmickael J., \& Jenkins J. C. (2012). Shifting Public Opinion on Climate Change: An Empirical Assessment of Factors Influencing Concern over Climate Change in the U.S., 2002- 
2010. Climatic Change, 114, 169-188. doi: 10.1007/s10584-012-0403-y.

Cook, J., Nuccitelli, D., Green, S. A., Richardson, M., Winkler, B., Painting, R.,Way, R., Jacobs, P., \& Skuce, A. (2013). Quantifying the consensus on anthropogenic global warming in the scientific literature. Environmental Research Letters, 8, 024024. doi:10.1088/17489326/8/2/024024

Corbett, J. B., \& Durfee, J. L. (2004). Testing public (un) certainty of science media representations of global warming. Science Communication, 26(2), 129-151. doi: $10.1177 / 1075547004270234$.

Farrell, J. (2016). Corporate funding and ideological polarization about climate change. Proceedings of the National Academy of Sciences, 113(1), 92-97.

Hu, S., \& Chen, J. (2016). Place-based inter-generational communication on local climate improves adolescents' perceptions and willingness to mitigate climate change. Climatic Change, 138(3), 425-238. doi:10.1007/s10584-016-1746-6.

Intergovernmental Panel on Climate Change (IPCC). (2014). Climate Change 2014: Synthesis Report. In: Pachauri, R. K., Meyer, L.A., \& Core Writing Team (eds.). Contribution of Working Groups I, II and III to the Fifth Assessment Report of the Intergovernmental Panel on Climate Change. IPCC, Genebra, Suiça.

Intergovernmental Panel on Climate Change (IPCC). (2007). Summary for Policymakers. In: Solomon, S., D. Qin, M. Manning, Z. Chen, M. Marquis, K.B. Averyt, M.Tignor \& H.L. Miller (eds.) Climate Change 2007: The Physical Science Basis. Contribution of Working Group I to the Fourth Assessment Report of the Intergovernmental Panel on Climate Change 2007. Inglaterra, Nova York, EUA: Cambridge University Press, Cambridge.

Jacobi, P. R., Guerra, A. F. S., Sulaiman, S. N., \& Nepomuceno, T. (2011). Mudanças climáticas globais: a resposta da educação. Revista Brasileira de Educação, 16(46). doi:10.1590/S141324782011000100008.

Kahn, M. E., \& Kotchen, M. J. (2010). Environmental concern and the business cycle: the chilling effect of recession. National Bureau of Economic Research, n.16241. doi:10.3386/w16241

Lorenzoni, I., \& Pidgeon, N. F. (2006). Public views on climate change: European and USA perspectives. Climatic Change, 77, 73-95. doi: 10.1007/s10584-006-9072-z.

Marshall, G. (2014). Don't Even Think About It: Why Our Brains Are Wired to Ignore Climate Change. New York: Bloomsbury.

Molion, L. C. B. (2008). Aquecimento global: uma visão crítica. Revista Brasileira de Climatologia, 3(4), 7-24. doi: 10.5380/abclima.v3i0.25404.

Moser, S. C. (2016). Reflections on climate change communication research and practice in the second decade of the 21st century: what more is there to say? Wiley Interdisciplinary Reviews: Climate Change, 7(3), 345-369. doi: 10.1002/wcc.403.

O'Neill, S., \& Boykoff, M. (2011). The role of new media in engaging the public with climate 
change. In: Whitmarsh, L., O'Neill, \& S., Lorenzoni, I. (Orgs.). Engaging the public with climate change: behaviour change and communication (pp. 233-251). USA: Routledge.

Posner, R. A. (2004). Catastrophe: risk and response. New York: Oxford University Press.

Sheppard, S. R. J. (2012). Visualizing climate change: a guide to visual communication of climate change and developing local solutions. New York: Routledge.

Silva, C. M. L. F. (2015). Mudanças climáticas e ambientais: contextos educacionais e históricos. Natal: Editora do IFRN.

Spence, A., Poortinga, W., \& Pidgeon, N. (2012). The psychological distance of climate change. Risk Analysis, 957-972.

Stoknes, P. E. (2015). What We Think About When We Try Not to Think About Global Warming: Toward a New Psychology of Climate Action. White River Junction, Vermont, Chelsea Green Books.

Stoutenborough, J. W., Fette, R. N., Vedlitz, A., \& Goldsmith, C. L. (2014). Understanding the Communication of Climate Change Risk: Climate Scientists' Perspectives of Media Sources and Policy Makers. Risk, Hazards \& Crisis in Public Policy, 5(4), 365-384.

Student Switch Off (SSO). (2016) The Student Switch Off. Recuperado de www.studentswitchoff.org.

Washington, H., \& Cook, J. (2011). Climate Change Denial: Heads in the Sand. London: Earthscan.

Watson, R. (org.), Carraro, C., Canziani, P., Nakicenovic, N., McCarthy, J. J., Goldemberg, J., Hisas, L. (2016). The Truth About Climate Change. Universal Ecological Fund - FED-US. Recuperado de http://feu-us.org/the-report/>.

Weber, E., \& Stern, P. (2011). Public understanding of climate change in the United States. American Psychologist, 66(4), 315-328.

World Resources Institute (WRI). (2015). Climate Analysis Indicators Tool (CAIT) 2.0: WRI's climate data explorer. Paris Contributions Map (INDCs). Recuperado de http://cait.wri.org/indc/.

Wozniak, A., Lück, J., \& Wessler, H. (2015). Frames, stories, and images: the advantages of a multimodal approach in comparative media content research on climate change. Environmental Communication, 9, 469-490.

Xue, W., Hine, D. W., Marks, A. D., Phillips, W. J., Nunn, P., \& Zhao, S. (2016). Combining threat and efficacy messaging to increase public engagement with climate change in Beijing, China. Climatic Change, 137, 43-55.

Zhao, X., Leiserowitz, A. A., Maibach, E. W., \& Roser-Renouf, C. (2011). Attention to science/environment news positively predicts and attention to political news negatively predicts global warming risk perceptions and policy support. Journal of Communication, 61(4), 713-31. 